\title{
THE MATHEMATICAL WORK OF MORGAN WARD
}

\author{
D. H. LEHMER
}

The mathematical works of Morgan Ward fall into seven categories as follows:

$\begin{array}{rll}\text { I } & \text { Recurring Series } & \text { (33 papers) } \\ \text { II } & \text { Diophantine Equations } & \text { ( } 8 \text { papers) } \\ \text { III } & \text { Abstract Arithmetic } & \text { (12 papers) } \\ \text { IV } & \text { Lattice Theory } & \text { (13 papers) } \\ \text { V } & \text { Functional Equations } & \text { (5 papers) } \\ \text { VI } & \text { Numerical Analysis } & \text { (4 papers) } \\ \text { VII } & \text { Miscellaneous } & \text { (7 papers) }\end{array}$

These seven topics do not represent seven periods of time. Early and late works are to be found in all categories. This is true especially of recurring series, a topic in which his sustained interest is most noticeable and one which I feel better qualified to discuss than any of the others.

The topic of recurring series belongs to the mixed additive-multiplicative theory of numbers. Each term of a recurring series being a fixed linear combination of its previous $k$ terms is additive in nature. Yet there may be unexpected divisibility and multiplicative properties to discover. For $k=1$ of course, we have essentially the successive powers of a given base, a truly multiplicative situation. But for $k=2$ we have the theory of Lucas' functions with their many elegant multiplicative properties and connections with cyclotomy. As with other kinds of problems, the going gets much harder when $k \geq 3$. It was one of Morgan Ward's major goals to describe the divisibility properties to be encountered, especially when $k=3$. At the same time he worked on the problem of strengthening the results known for $k=2$. Indeed his last paper adds to our knowledge of classes of primes dividing the ancient series of Fibonacci, see [80]. Other papers for $k=2$ are concerned with the so-called intrinsic divisors of $U_{n}=\left(\alpha^{n}-\beta^{n}\right) /(\alpha-\beta)$, where $\alpha, \beta$ are the roots of $X^{2}-R X+Q=0$, namely those primes $p$ which divide $U_{n}$ but not $U_{m}$ for $m<n$. The theorems are to the effect that every $U_{n}$ has such a prime divisor except in a finite number of specified choices $(R, Q)$ and then for only specified values of $n$. The celebrated Fibonacci number $F_{12}=144$ is an exceptional case in point.

Received by the editor August 3, 1992.

1991 Mathematics Subject Classification. Primary 01A70, 11-03.

Editorial note. This paper is based on a handwritten manuscript discovered in the Morgan Ward file of D. H. Lehmer's reprint collection. It seems to have been prepared for a talk made at the Caltech Symposium in memory of Morgan Ward, November 21-22, 1963. In 1930, D. H. and Emma Lehmer had come to Caltech, where the Wards were among their earliest friends. This paper reflects D. H. Lehmer's respect for, and appreciation of, the mathematical work of others. 
Results like these have practical value in proving theorems on the distribution of power residues.

Another subject of study that intrigued Ward was the so-called divisibility sequences of Marshall Hall, namely sequences $U_{n}$ such that $a \mid b$ implies that $U_{a} \mid U_{b}$. Many second-order recurring series are divisibility sequences as well as some recurring series of higher order. Ward $[60,62,65,66]$ found other kinds of divisibility sequences associated with the theory of the real multiplication of Jacobi elliptic functions.

Of the many results about recurrences of order $k=3$ we select one that is easy to state. Let $W_{1}, W_{2}, W_{3}, \ldots$ be nonperiodic and satisfy $W_{k+1}=P W_{k}-$ $Q W_{k-1}+R W_{k-2}$, and suppose that $X^{3}-P X^{2}+Q X-R=(X-a)(X-b)(X-c)$, where $a, b$ and $c$ are coprime integers. Then $W_{r}=0$ for at most 3 values of $r$. Since $W_{n}=A a^{r}+B b^{r}+C c^{r}$, this result is something like Fermat's Last Theorem.

On Diophantine equations, two problems may be cited.

1. Multiplicative Diophantine systems. The system

$$
X^{9}=Y^{5}=U^{4} V^{4}=W R S T
$$

has a parametric solution involving 46127626 parameters, see [18].

2. Euler's Conjecture: ${ }^{1} X^{4}+Y^{4}+Z^{4}=W^{4}$ has no nontrivial solution. In [59] Ward showed that any nontrivial solution must have $W>10^{4}$. This was done by hand calculation in 1945 .

Ward's abstract arithmetic papers comprise a variety of notes on subjects ranging over group theory, logic, and algebraic number theory.

Morgan Ward had unbounded enthusiasm for the work of others that appealed to his sense of beauty. In many cases he proceeded to dig in these other fields and to help uncover their treasures. It was thus that he became enthused about Garrett Birkhoff's lattice theory. Of his many papers on the subject most were concerned with so-called residuated lattices.

Ward's functional equation papers include two on the problem of continuous iteration. Let $E(x)>x$ be continuous and steadily increasing. Write $E_{0}(x) \equiv$ $x, E_{1}(x)=E(x), E_{2}(x)=E(E(x))$, etc. How can you define $E_{y}(x)$ for nonintegral $y$ ? Ward found that the most general solution of the problem is

$$
E_{y}(x)=\psi\left(\psi^{-1}(x)+y\right),
$$

where $\psi(x)=E_{[x]}(\theta(x-[x]))$ and $\theta$ is any continuous function that increases steadily from $\theta(0)=0$ to $\theta(1)=1$, see [36]. Other papers deal with the properties of the coefficients of power series of functions $E(x)$ for which $E_{2}(x)=x$, with reversion of power series, and with special Appel polynomials.

Numerical analysis papers are mainly notes on the practical calculation of special functions and integrals. Some of the work he did during World War II gave him a chance to consider such matters. Actually almost all of his papers are full of examples of what he is talking about. He was also an exponent of the experimental approach to research. When his ingenuity was insufficient to solve all cases of a difficult problem he never was too proud to point this out.

${ }^{1}$ Editorial note. Euler's Conjecture was recently shown to be false by Noam Elkies, Math. Comp. 51 (1988), 825-835. The minimal counterexample was found by Roger Frye, and is $95800^{4}+$ $217519^{4}+414560^{4}=422481^{4}$. 
I have mentioned his excitable and contagious enthusiasm for the fine work of many other writers. On the other hand, like E. T. Bell, he had great contempt for those who proliferate easy empty generalizations of the great classic ideas of mathematics.

\section{RESEARCH PUBLICATIONS OF MORgan WARD}

1. A generalization of recurrents, Bull. Amer. Math. Soc. 33 (1927), 477-492.

2. General arithmetic, Proc. Nat. Acad. Sci. U.S.A. 13 (1927), 748-749.

3. Postulates for an abstract arithmetic, Proc. Nat. Acad. Sci. U.S.A. 14 (1928), 907-911.

4. A simplification of certain problems in arithmetical division, Amer. Math. Monthly 35 (1928), 9-14.

5. On certain functional relations, Amer. Math. Monthly 36 (1929), 426-431.

6. Certain expansions involving doubly infinite series, Ann. of Math. (2) 30 (1929), 578-582.

7. A certain class of polynomials, Ann. of Math. (2) 31 (1930), 43-51.

8. A generalization of a familiar theorem concerning prime numbers, J. London Math. Soc. 5 (1930), 106-107.

9. The reversion of a power series, Rend. Circ. Mat. Palermo 54 (1930), 42-46.

10. Postulates for the inverse operations in a group, Trans. Amer. Math. Soc. 32 (1930), 520-526.

11. The characteristic number of a sequence of integers satisfying a linear recursion relation, Trans. Amer. Math. Soc. 33 (1931), 153-165.

12. The algebra of recurring series, Ann. of Math. (2) 32 (1931), 1-9.

13. Some arithmetical properties of sequences satisfying a linear recursion relation, Ann. of Math. (2) 32 (1931), 734-738.

14. The distribution of residues in a sequence satisfying a linear recursion relation, Trans. Amer. Math. Soc. 33 (1931), 166-190.

15. Conditions for the solubility of the diophantine equation $x^{2}-M y^{2}=-1$, Trans. Amer. Math. Soc. 33 (1931), 712-718.

16. The linear form of numbers represented by a homogeneous polynomial in any number of variables, Ann. of Math. (2) 33 (1932), 324-326.

17. On the behaviour of non-static models of the universe when the cosmological term is omitted, (with R. C. Tolman), Phys. Rev. 39 (1932), 835-843.

18. A type of multiplicative diophantine system, Amer. J. Math. 55 (1933), 67-76.

19. A property of recurring series, Proc. Nat. Acad. Sci. U.S.A. 19 (1933), 914-916.

20. The cancellation law in the theory of congruences to a double modulus, Trans. Amer. Math. Soc. 35 (1933), 254-260.

21. The arithmetical theory of linear recurring series, Trans. Amer. Math. Soc. 35 (1933), $600-$ 628.

22. A certain class of trigonometric integrals, Amer. Math. Monthly 40 (1933), 340-346.

23. The representation of Stirling's numbers and Stirling's polynomials as sums of factorials, Amer. J. Math. 56 (1934), 87-95.

24. On the vanishing of the sum of the $N^{\text {th }}$ powers of the roots of a cubic equation, Amer. Math. Monthly 41 (1934), 313-316.

25. Note on the period of a mark in a finite field, Bull. Amer. Math. Soc. 40 (1934), 279-281.

26. Note on an arithmetical property of recurring series, Math. Z. 39 (1934), 211-214.

27. An arithmetical property of recurring series of the second order, Bull. Amer. Math. Soc. 40 (1934), 825-828.

28. Note on the iteration of functions of one variable, Bull. Amer. Math. Soc. 40 (1934), 688-690.

29. The numerical evaluation of a class of trigonometric series, Amer. Math. Monthly 41 (1934), 563-565. 
30. An enumerative problem in the arithmetic of linear recurring series, Trans. Amer. Math. Soc. 37 (1935), 435-440.

31. A determination of all possible systems of strict implication, Amer. J. Math. 57 (1935), 261-266.

32. Conditions for factorization in a set closed under a single operation, Ann. of Math. (2) 36 (1935), 36-39.

33. On the factorization of polynomials to a prime modulus, Ann. of Math. (2) 36 (1935), 870874.

34. The diophantine equation $X^{2}-D Y^{2}=Z^{m}$, Trans. Amer. Math. Soc. 38 (1935), 447-457.

35. A calculus of sequences, Amer. J. Math. 58 (1936), 255-266.

36. The continuous iteration of real functions, Bull. Amer. Math. Soc. 42 (1936), 393-396.

37. The null divisors of linear recurring series, Duke Math. J. 2 (1936), 472-476.

38. Note on divisibility sequences, Bull. Amer. Math. Soc. 42 (1936), 843-845.

39. Linear divisibility sequences, Trans. Amer. Math. Soc. 41 (1937), 276-286.

40. Arithmetic functions on rings, Ann. of Math. (2) 38 (1937), 725-732.

41. Some arithmetical applications of residuation, Amer. J. Math. 59 (1937), 921-926.

42. Residuation in structures over which a multiplication is defined, Duke Math. J. 3 (1937), 627-636.

43. Arithmetical properties of sequences in rings, Ann. of Math. (2) 39 (1938), 210-219.

44. Residuated lattices, Proc. Nat. Acad. Sci. U.S.A. 24 (1938), 162-164.

45. The law of apparition of primes in a Lucasian sequence, Trans. Amer. Math. Soc. 44 (1938), 68-86.

46. Structure residuation, Ann. of Math. (2) 39 (1938), 558-568.

47. A note on divisibility sequences, Bull. Amer. Math. Soc. 45 (1939), 334-336.

48. Evaluations over residuated structures (with R. P. Dilworth), Ann. of Math. (2) 40 (1939), 328-338.

49. Residuated lattices (with R. P. Dilworth), Trans. Amer. Math. Soc. 45 (1939), 335-354.

50. The algebra of lattice functions, Duke Math. J. 5 (1939), 357-371.

51. Ring homomorphisms which are also lattice homomorphisms, Amer. J. Math. 61 (1939), 783-787.

52. A characterization of Dedekind structures, Bull. Amer. Math. Soc. 45 (1939), 448-451.

53. Note on the general rational solution of the equation $a x^{2}-b y^{2}=c z^{3}$, Amer. J. Math. 61 (1939), 788-790.

54. The lattice theory of ova (with R. P. Dilworth), Ann. of Math. (2) 40 (1939), 600-608.

55. A characterization of Boolean algebras (with Garrett Birkhoff), Ann. of Math. (2) 40 (1939), 609-610.

56. Residuated distributive lattices, Duke Math. J. 6 (1940), 641-651.

57. The arithmetical properties of modular lattices, Rev. Ci. Lima 430 (1941), 593-603.

58. The closure operators of a lattice, Ann. of Math. (2) 43 (1942), 191-196.

59. Euler's three biquadrate problem, Proc. Nat. Acad. Sci. U.S.A. 31 (1945), 125-127.

60. Memoir on elliptic divisibility sequences, Amer. J. Math. 70 (1948), 31-74.

61. Euler's problem on sums of three fourth powers, Duke Math. J. 15 (1948), 827-837.

62. The law of repetition of primes in an elliptic divisibility sequence, Duke Math. J. 15 (1948), 941-946.

63. A generalized integral test for convergence of series, Amer. Math. Monthly 56 (1949), 170172.

64. Note on a paper by C. E. Dickart, R. P. Dilworth and Morgan Ward, Bull. Amer. Math. Soc. 55 (1949), 1141.

65. Arithmetical properties of the elliptic polynomials arising from the real multiplication of the Jacobi functions, Amer. J. Math. 72 (1950), 284-302. 
66. Arithmetical properties of polynomials associated with lemniscate elliptic functions, Proc. Nat. Acad. Sci. U.S.A. 36 (1950), 359-362.

67. A class of soluble diophantine equations, Proc. Nat. Acad. Sci. U.S.A. 37 (1951), 113-114.

68. Prime divisors of second order recurring series, Duke Math. J. 21 (1954), 607-614.

69. The maximal prime divisors of linear recurrences, Canad. J. Math. 6 (1954), 455-462.

70. Cyclotomy and the converse of Fermat's theorem, Amer. Math. Monthly 61 (1954), 564.

71. The intrinsic divisors of Lehmer numbers, Ann. of Math. (2) 62 (1955), 230-236.

72. On the number of vanishing terms in an integral cubic recurrence, Amer. Math. Monthly 62 (1955), 155-160.

73. The law of apparition and repetition of primes in a cubic sequence, Trans. Amer. Math. Soc. 79 (1955), 72-90.

74. The mappings of the positive integers into themselves which preserve division, Pacific J. Math. 5 (1955), 1013-1023.

75. Tests for primality based on Sylvester's cyclotomic numbers, Pacific J. Math. 9 (1959), 12691272.

76. The vanishing of the homogeneous product sum of the roots of a cubic, Duke Math. J. 26 (1959), 553-662.

77. Some diophantine problems connected with linear recurrences, Report of the Institute in the Theory of Numbers, Univ. Colorado, Boulder, Colorado, 1959, pp. 250-257.

78. The vanishing of the homogeneous product sum of three letters, Duke Math. J. 27 (1960), 619-624.

79. The calculation of the complete elliptic integral of the third kind, Amer. Math. Monthly 67 (1960), 205-213.

80. The prime divisors of Fibonacci numbers, Pacific J. Math. 11 (1961), 379-386.

81. The linear p-adic recurrence of order two, Illinois J. Math. 6 (1962), 40-52.

82. Divisors of recurrent sequences (with E. C. Dade, D. W. Robinson, O. Taussky), J. Reine Angew. Math. 214/215 (1964), 180-183.

Department of Mathematics, University of California at Berkeley, Berkeley, California 94720 